\title{
Characterization of Aggregate Interference in Arbitrarily-shaped Underlay Cognitive Networks
}

\author{
Jing Guo, Salman Durrani and Xiangyun Zhou \\ Research School of Engineering, College of Engineering and Computer Science, \\ The Australian National University, Canberra, ACT 0200, Australia. \\ Emails: \{jing.guo, salman.durrani, xiangyun.zhou\}@anu.edu.au.
}

\begin{abstract}
This paper characterizes the aggregate interference at the primary user (PU) due to $M$ secondary users (SUs) in an underlay cognitive network, where appropriate SU activity protocols are employed in order to limit the interference generated by the SUs. Different from prior works, we assume that the PU can be located anywhere inside an arbitrarilyshaped convex network region. Using the moment generating function (MGF) of the interference from a random SU, we derive general expressions for the $n$-th moment and the $n$ th cumulant of the aggregate interference for guard zone and multiple-threshold SU activity protocols. Using the cumulants, we study the convergence of the distribution of the aggregate interference to a Gaussian distribution. In addition, we compare the well-known closed-form distributions in the literature to approximate the complementary cumulative distribution function (CCDF) of the aggregate interference. Our results show that care must be undertaken in approximating the aggregate interference as a Gaussian distribution, even for a large number of SUs, since the convergence is not monotonic in general. In addition, the shifted lognormal distribution provides the overall best CCDF approximation, especially in the distribution tail region, for arbitrarily-shaped network regions.
\end{abstract}

\section{INTRODUCTION}

Cognitive radio network is a promising technology for $5 \mathrm{G}$ wireless communication networks [1]. In cognitive networks, the unlicensed secondary users (SUs) are allowed dynamic and opportunistic access to the licensed spectrum band allocated to the primary users (PUs) [2]. Based on the access technology of the licensed spectrum sharing, the cognitive networks can be classified into underlay, overlay and interweave networks [3]. In this paper, we focus on underlay cognitive networks in which the SUs coexist with the PU in the licensed band by keeping the interference power at PU under a certain limit. Hence, the characterization of the aggregate interference from the SUs is a crucial problem in underlay cognitive networks.

Recently, the characterization of the aggregate interference in underlay cognitive networks has received attention in the literature [4-7]. Considering an infinite cognitive network region, the closed-form bounds for the moment generating function (MGF) of the aggregate interference were derived in [4]. Considering the PU to be located at the center of an annulus-shaped region, the MGF and mean of the aggregate interference were derived in [5] and the cumulants of the aggregate interference were derived in [6]. For a disk region with arbitrary location of PU and Rayleigh fading channels, the MGF and cumulants of the aggregate interference were derived in [7]. However, in practice, the shape of the network can be arbitrary.

Using the cumulants of the aggregate interference, the distribution of the aggregate interference can be approximated by the well known distributions [8, Technique 3]. In this context, for wireless ad-hoc networks, the aggregate interference from a large number of interfering nodes was approximated as a Gaussian variable in [9]. However, as shown in [9], when the number of interfering node is moderate, the kurtosis is not close to zero and the Gaussian distribution does not provide a good approximation. As alternatives, the inverse Gaussian distribution was proposed in [10] and the lognormal distribution was proposed in [11]. For interweave cognitive networks, it was shown in [12] that the lognormal distribution increased the approximation error in the distribution tail region. Hence the shifted lognormal distribution was proposed in [12] and the Gamma distribution was proposed in [13] for Nakagami fading channels. For underlay cognitive networks, which are the focus of this paper, the truncated stable distribution was proposed in [6]. However, it does not have a closed-form representation in general and is determined by its characteristic function. To best of authors' knowledge, a comparison of the different distributions to approximate the aggregate interference distribution in arbitrarily-shaped underlay cognitive networks has not been addressed in the literature to date.

In this paper, we focus on characterizing the aggregate interference in arbitrarily-shaped underlay cognitive network regions with arbitrary location of the PU. We consider both the guard zone protocol $[4,5]$ and the multi-threshold protocol (which includes the single-threshold protocol as a special case) [6], which are SU activity protocols commonly employed in underlay cognitive networks in order to limit the interference generated by SUs. Compared to prior works, the major contributions of this paper are:

- We derive the analytical expressions for the $n$-th moment and the $n$-th cumulant of the aggregate interference from the SUs for the guard zone and multi-threshold protocols. We show that existing $n$-th moment results in the literature arise as special cases from our general expressions.

- We study the convergence of the aggregate interference distribution to a Gaussian distribution via the higher order statistics (i.e., skewness and kurtosis). We show that caution must be taken in using the Gaussian approximation, 
even for a large number of SUs, as the rate of convergence to a Gaussian depends on the path-loss exponent, the fading and the parameters of SU activity protocols and the convergence is in general non-monotonic.

- We compare the different closed-form distributions available in the literature to approximate the CCDF of the aggregate interference in arbitrarily-shaped convex regions. We show that the shifted lognormal distribution provides the overall best approximation, especially in the distribution tail region.

The rest of this paper is organized as follows. Section II describes the system model, including the SU activity protocols. The general $n$-th cumulant expressions to characterize the aggregate interference are derived in Section III. The statistical tools used to study the distribution of aggregate interference are discussed in Section IV. The numerical and simulation results are provided in Section V. Finally Section VI concludes the paper.

\section{SYSTEM MODEL}

We consider an underlay cognitive network with a primary user and $M$ secondary users independently and uniformly distributed (i.u.d.) inside an arbitrarily-shaped convex region $\mathcal{A}$, in the two dimensional Euclidean space $\mathbb{R}^{2}$. The interfered PU can be located anywhere inside this network region $\mathcal{A}$, as depicted in Fig. 1. Under this setup, the distance between $i$-th $\mathrm{SU}$ and $\mathrm{PU}$, denoted as $R_{i}$, is a random variable (RV) with probability distribution function (PDF) $f_{R_{i}}\left(r_{i}\right)$. For any irregular convex polygon region with an arbitrarily-located reference point, the distance distribution $f_{R_{i}}\left(r_{i}\right)$ and the corresponding cumulative distribution function (CDF) $F_{R_{i}}\left(r_{i}\right)$ can be determined in closed-form using the algorithm in [14, 15] and the accompanying software implementation [16].

We assume that all the transmitting channels are identically and independently distributed (i.i.d.). We model the wireless channel as the path-loss plus block fading channel, i.e., the propagation loss at distance $r$ can be expressed as $G l(r)$, where $G$ denotes the fading power gain on the channel and $l(r)=\min \left\{1, r^{-\alpha}\right\}$ is the bounded path-loss model with pathloss exponent $\alpha$, which avoids the singularity at $r=0$ [17].

We assume that all the nodes are in the frequency duplex mode. Similar to [6], to know the channel strength to the PU, each SU senses the PU's uplink channel. In other words, the sensing is achieved by receiving the signal transmitted by the PU for each SU via the sensing channel (from PU to SU). The sensing channel is assumed to be fully uncorrelated with the SU transmitting (i.e., interfering) channel (from SU to PU).

Each SU determines whether to transmit or not according to the adopted SU activity protocol. Our purpose is to characterize the statistics of aggregate interference from the $M$ SUs at the given location of the PU, while taking into account the SU activity protocol. We can express the aggregate interference at $\mathrm{PU}$ as

$$
I_{\mathrm{agg}}=\sum_{i=1}^{M} I_{i}=\sum_{i=1}^{M} P_{T} G_{i} l\left(R_{i}\right) \mathbf{1}_{(\text {condition })},
$$

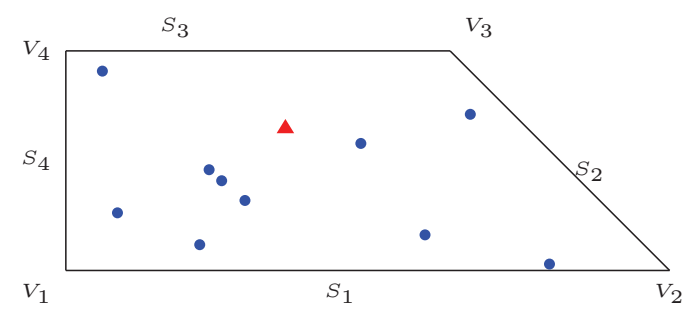

Fig. 1. Illustration of an arbitrarily-shaped underlay cognitive network region $(\boldsymbol{\Delta}=$ interfered PU, $\bullet=\mathrm{SU}) . S_{j}$ and $V_{j}(j=1,2,3,4)$ denote the side and vertex of this convex shape.

where $I_{i}$ is the interference generated by the $i$-th $\mathrm{SU}, l\left(R_{i}\right)=$ $\min \left\{1, R_{i}^{-\alpha}\right\}, P_{T}$ denotes the transmit power for SU, which is assumed to be the same for all SUs, and $G_{i}$ represents the instantaneous fading power gain on the SU transmitting channel with PDF $f_{G_{i}}\left(g_{i}\right)$. $\mathbf{1}_{\text {(condition) }}$ is an indicator function which is equal to one as long as the condition within it, which depends on the SU activity protocol, holds. In the following, we consider the two important SU activity protocols for the underlay cognitive networks and formulate the explicit expression for $I_{\mathrm{agg}}$ for each protocol ${ }^{1}$.

\section{A. Guard Zone Protocol}

In this protocol, the PU is protected by a guard zone of radius $r_{f}$ around itself $[4,5]$. Any SU that enters this region becomes inactive and does not transmit, i.e., no interference is generated from the SUs inside the guard zone. Then the aggregate interference from SUs is given by

$$
I_{\mathrm{agg}}=\sum_{i=1}^{M} P_{T} G_{i} R_{i}^{-\alpha} \mathbf{1}_{\left(R_{i}>r_{f}\right)} .
$$

\section{B. Multi-Threshold Protocol}

For the multi-threshold protocol, each SU adjusts its transmit power based on the received instantaneous power on the sensing channel [6]. The sensed signal power at the $i$-th SU is given by $P_{T_{s}} H_{i} l\left(R_{i}\right)$, where $P_{T_{s}}$ denotes the transmit power for PU and has been set to unity for analytical convenience.

Assume that there are $L$ level of transmit power and let $\gamma_{l-1}$ $(l=1, \ldots, L)$ and $\gamma_{l}$ denote the lower and upper threshold for a certain transmit power level $l$, respectively. When the sensed power lies in the range of $\gamma_{l-1}$ and $\gamma_{l}$, the SU transmits the power at level $l$, denoted by $P_{(T, l)}$. Mathematically, the transmit power for the $i$-th $\mathrm{SU}$ is

$$
P_{T}= \begin{cases}P_{(T, 1)}, & \gamma_{0} \leq H_{i} l\left(R_{i}\right)<\gamma_{1} \\ \cdots & \cdots \\ P_{(T, L)}, & \gamma_{L-1} \leq H_{i} l\left(R_{i}\right) \leq \gamma_{L}\end{cases}
$$

Throughout the paper, we assume that $\gamma_{0}=0$ and $\gamma_{L}=\infty$.

Note that when $P_{(T, L)}$ is set to zero, the SU becomes inactive once its received power on the sensing channel is greater than the threshold $\gamma_{L-1}$. Additionally, for the case of two power levels $(L=2)$ and the power for the second level

\footnotetext{
${ }^{1}$ The detailed performance comparison of different secondary user activity protocols has been addressed in [18].
} 
being zero $\left(P_{(T, 2)}=0\right)$, the multi-threshold protocol reduces to the single-threshold protocol [6].

For the above setup, the aggregate interference from SUs can be written as

$$
I_{\text {agg }}=\sum_{i=1}^{M} \sum_{l=1}^{L} P_{(T, l)} G_{i} R_{i}^{-\alpha} \mathbf{1}_{\left(\gamma_{l-1} \leq H_{i} l\left(R_{i}\right)<\gamma_{l}\right)} .
$$

\section{AgGregate InTERFERENCE}

In this section, we provide the mathematical formulation to characterize the aggregate interference from SUs under the considered SU activity protocols.

\section{A. Mathematical Formulation}

Since the SUs are i.u.d. inside the finite network region, the moment generating function of $I_{\text {agg }}$ is given by

$$
\mathcal{M}_{I_{\text {agg }}}(s)=\mathbb{E}_{I_{\text {agg }}}\left\{\exp \left(-s I_{\text {agg }}\right)\right\}=\left\{\mathcal{M}_{I}(s)\right\}^{M},
$$

where $\mathbb{E}_{I_{\text {agg }}}\{\cdot\}$ denotes the expectation with respect to the $\mathrm{RV} I_{\text {agg }}$ and $\mathcal{M}_{I}(s)=\mathbb{E}_{I}\{\exp (-s I)\}$ is the MGF of the interference from a random SU. Note that we have dropped the index $i$ in $I_{i}$ (similarly, in $R_{i}, G_{i}$ and $H_{i}$ ) because of the assumptions that the SUs are i.u.d. and the fading channels are i.i.d..

Using its definition, the $n$-th cumulant of $I_{\text {agg }}$ is related to the MGF of $I_{\text {agg }}$ by [19]

$$
\begin{aligned}
\kappa_{I_{\text {agg }}}(n) & =\left.(-1)^{n} \frac{d^{n} \ln \mathcal{M}_{I_{\text {agg }}}(s)}{d s^{n}}\right|_{s=0} \\
& =\left.(-1)^{n} M \frac{d^{n} \ln \mathcal{M}_{I}(s)}{d s^{n}}\right|_{s=0}=M \kappa_{I}(n),
\end{aligned}
$$

where $\kappa_{I}(n)$ denotes the $n$-th cumulant of the interference from a random SU.

As it is not easy to directly evaluate $\kappa_{I}(n)$ for $n>2$, we compute the $n$-th moment of the interference from a random SU, denoted by $\mu_{I}(n)$, instead. Using the recursive momentcumulant relationship [9], we can rewrite (6) as

$$
\kappa_{I_{\text {agg }}}(n)=M\left(\mu_{I}(n)-\sum_{j=1}^{n-1}\left(\begin{array}{c}
n-1 \\
j-1
\end{array}\right) \kappa_{I}(j) \mu_{I}(n-j)\right)
$$

and $\mu_{I}(n)$ is related to $\mathcal{M}_{I}(s)$ by [19]

$$
\mu_{I}(n)=\left.(-1)^{n} \frac{d^{n} \mathcal{M}_{I}(s)}{d s^{n}}\right|_{s=0} .
$$

In the following, we present analytical results for $\mu_{I}(n)$ for the two SU activity protocols considered in this paper.

\section{B. Guard Zone Protocol}

For the guard zone protocol, the interference from a random SU is $I=P_{T} G l(R) \mathbf{1}_{\left(R>r_{f}\right)}$. Using the definition of MGF, the MGF of $I$ is given by

$$
\begin{aligned}
\mathcal{M}_{I}(s) & =\mathbb{E}_{G, R}\left\{\exp \left(-s P_{T} G l(R) \mathbf{1}_{\left(R>r_{f}\right)}\right)\right\} \\
& =\int_{0}^{\infty} \int_{r_{f}}^{r_{\max }} \exp \left(-s P_{T} G l(R)\right) f_{G}(g) f_{R}(r) d r d g,
\end{aligned}
$$

where $r_{\max }$ denotes the maximum range of the RV $R$ and $\mathbb{E}_{G, R}\{\cdot\}$ denotes the expectation with respect to RVs $G, R$.

Substituting (9) into (8), the $n$-th moment of $I$ can be computed as

$$
\begin{aligned}
\mu_{I}(n)= & \left(P_{T}\right)^{n} \int_{0}^{\infty} g^{n} f_{G}(g) d g \int_{r_{f}}^{r_{\max }}(l(R))^{n} f_{R}(r) d r \\
= & P_{T}^{n} \mathbb{E}_{G}\left\{G^{n}\right\}\left(\left(F_{R}(1)-F_{R}\left(r_{f}\right)\right) \mathbf{1}_{\left(r_{f}<1\right)}\right. \\
& \left.+\int_{\max \left(1, r_{f}\right)}^{r_{\max }} r^{-n \alpha} f_{R}(r) d r\right),
\end{aligned}
$$

where $F_{R}(\cdot)$ and $f_{R}(r)$ are the CDF and PDF of the distance from a $\mathrm{SU}$ to the PU.

Remark 1: For the guard zone protocol, the $n$-th moment expressed in (10) generally admits the closed-form solution. Note that $\mathbb{E}_{G}\left[G^{n}\right]$ represents the $n$-th moment for the fading power gain, for which the closed-form expression exists for most common fading distributions. For example, when the transmitting channel undergoes the Nakagami- $m$ fading with the fading coefficient $m_{g}, \mathbb{E}_{G}\left[G^{n}\right]=\frac{\left(m_{g}+n-1\right) !}{m_{g}^{n}\left(m_{g}-1\right) !}$ [9]. Similarly, the integration involving $f_{R}(r)$ can be computed in closed-form once $f_{R}(r)$ is determined using [16].

\section{Multi-Threshold Protocol}

For the multi-threshold protocol, as the interference is $I=$ $\sum_{l=1}^{L} P_{(T, l)} G R^{-\alpha} \mathbf{1}_{\left(\gamma_{l-1} \leq H l(R)<\gamma_{l}\right)}$, the conditional probability mass function of the interference, which is conditioned on the distance $R$, can be written as

$$
\operatorname{Pr}(I=\mathbb{I} \mid R)= \begin{cases}\int_{0}^{\gamma_{1} / l(R)} f_{H}(h) d h, & \mathbb{I}=P_{(T, 1)} G l(R) ; \\ \int_{\gamma_{1} / l(R)}^{\gamma_{2} / l(R)} f_{H}(h) d h, & \mathbb{I}=P_{(T, 2)} G l(R) ; \\ \ldots & \ldots \\ \int_{\gamma_{L-1} / l(R)}^{\infty} f_{H}(h) d h, & \mathbb{I}=P_{(T, L)} G l(R) ;\end{cases}
$$

Thus, we can write the MGF of the interference from a random $\mathrm{SU}$ as

$$
\begin{aligned}
\mathcal{M}_{I}(s)= & \mathbb{E}_{G}\left\{\mathbb{E}_{R}\{\operatorname{Pr}(I=\mathbb{I} \mid R) \exp (-s \mathbb{I})\}\right\} \\
= & \sum_{l=1}^{L} \int_{0}^{\infty} \int_{0}^{r_{\max }} \exp \left(-s P_{(T, l)} G l(R)\right) \times \\
& \left(F_{H}\left(\gamma_{l} / l(R)\right)-F_{H}\left(\gamma_{l-1} / l(R)\right)\right) f_{R}(r) f_{G}(g) d r d g .
\end{aligned}
$$

Substituting (12) into (8), $\mu_{I}(n)$ is expressed as

$$
\begin{aligned}
\mu_{I}(n)= & \int_{0}^{\infty} g^{n} f_{G}(g) d g \sum_{l=1}^{L} P_{(T, l)}^{n} \times \\
& \int_{0}^{r_{\max }}(l(R))^{n}\left(F_{H}\left(\gamma_{l} / l(R)\right)-F_{H}\left(\gamma_{l-1} / l(R)\right)\right) f_{R}(r) d r \\
= & \mathbb{E}_{G}\left[G^{n}\right] \sum_{l=1}^{L} P_{(T, l)}^{n}\left(\left(F_{H}\left(\gamma_{l}\right)-F_{H}\left(\gamma_{l-1}\right)\right) F_{R}(1)+\right. \\
& \left.\int_{1}^{r_{\max }} r^{-n \alpha}\left(F_{H}\left(\gamma_{l} r^{\alpha}\right)-F_{H}\left(\gamma_{l-1} r^{\alpha}\right)\right) f_{R}(r) d r\right)
\end{aligned}
$$




$$
\begin{aligned}
\mu_{I}(n)= & \frac{\left(m_{g}+n-1\right) !}{m_{g}^{n}\left(m_{g}-1\right) !} \frac{2}{W^{2}(2-n \alpha)}\left(\sum _ { l = 1 } ^ { L - 1 } ( P _ { ( T , l ) } ^ { n } - P _ { ( T , l + 1 ) } ^ { n } ) \left(W^{2-n \alpha} \frac{\Gamma\left[m_{h}, 0, m_{h} \gamma_{l} W^{\alpha}\right]}{\Gamma\left[m_{h}\right]}-\frac{n \alpha}{2} \frac{\Gamma\left[m_{h}, 0, m_{h} \gamma_{l}\right]}{\Gamma\left[m_{h}\right]}\right.\right. \\
& \left.\left.-\left(m_{h} \gamma_{l}\right)^{n-\frac{2}{\alpha}} \frac{\Gamma\left[m_{h}-n+\frac{2}{\alpha}, m_{h} \gamma_{l}, m_{h} \gamma_{l} W^{\alpha}\right]}{\Gamma\left[m_{h}\right]}\right)+P_{(T, L)}^{n}\left(W^{2-n \alpha}-\frac{n \alpha}{2}\right)\right) .
\end{aligned}
$$

where $F_{H}(\cdot)$ denotes the $\mathrm{CDF}$ of fading power gain on the sensing channel.

Special Case: When the PU is located at the center of a disk with radius $W$, we have $f_{R}(r)=2 r / W^{2}$. Assuming all the fading channels experience the Nakagami- $m$ fading, we can derive the closed-form result for the $n$-th moment given in (14), where $n \alpha \neq 2, m_{g}$ and $m_{h}$ represent the fading parameter on SU transmitting channel and sensing channel respectively, $\Gamma[x]=\int_{0}^{\infty} t^{x-1} \exp (-t) d t$ and $\Gamma[x, a, b]=$ $\int_{a}^{b} t^{x-1} \exp (-t) d t$ are the complete and incomplete gamma functions, respectively [19]. The result from (14) is the same as the result from [6] with the same system model. However, the method of deriving the $n$-th moment in [6] is only valid for the annulus-shaped region with PU located at its center.

\section{Characterization of the Aggregate INTERFERENCE}

In this section, we describe the statistical tools which can be used to characterize the aggregate interference.

\section{A. Skewness, Kurtosis and Convergence to a Gaussian}

The skewness and kurtosis describe the 'symmetry' and 'peakedness' of the distribution of a RV, respectively. The skewness, $\beta_{1}$, and kurtosis, $\beta_{2}$, of a RV $X$ are related to its $n$-th cumulant by [19]

$$
\beta_{1}(X)=\frac{\kappa_{X}(3)}{\kappa_{X}(2)^{3 / 2}},
$$

and

$$
\beta_{2}(X)=\frac{\kappa_{X}(4)}{\kappa_{X}(2)^{2}} .
$$

Together, the skewness and kurtosis can be employed to assess the normality of a distribution [20]. For a Gaussian distribution, $\beta_{1}=\beta_{2}=0$. Thus, if both $\beta_{1}(X) \rightarrow 0$ and $\beta_{2}(X) \rightarrow 0$, we can say that the RV $X$ is closely approximated by a Gaussian distribution [9].

\section{B. Distribution Approximation of the Aggregate Interference}

The exact $n$-th cumulant of $I_{\text {agg }}$ can be derived by substituting (10) and (13), respectively, into (7) for the considered SU activity protocols. Once $\kappa_{I_{\mathrm{agg}}}(n)$ is known, its distribution can be approximated by the well known closed-form distributions. In this section, we detail the various distributions which can be used to approximate the complementary cumulative distribution function (CCDF) of $I_{\mathrm{agg}}$. Their accuracy is compared in Section V.
Gaussian distribution: The CCDF of Gaussian distribution is [19]

$$
\operatorname{Pr}\left(I_{\mathrm{agg}}>z\right)^{\text {Gaussian }}=Q\left(\frac{z-\kappa_{I_{\mathrm{agg}}}(1)}{\sqrt{\kappa_{I_{\mathrm{agg}}}(2)}}\right),
$$

where $Q(\cdot)$ is the Q-function, $\kappa_{I_{\text {agg }}}(1)$ and $\kappa_{I_{\text {agg }}}(2)$ are 1 st and 2nd cumulant of $I_{\mathrm{agg}}$, respectively.

Inverse Gaussian distribution: The CCDF of Inverse Gaussian distribution is [19]

$$
\begin{aligned}
& \operatorname{Pr}\left(I_{\text {agg }}>z\right)^{\text {inverse Gaussian }}=Q\left(\sqrt{\frac{\kappa_{I_{\text {agg }}}(1)^{3}}{\kappa_{I_{\text {agg }}}(2) z}}\left(\frac{z}{\kappa_{I_{\text {agg }}}(1)}-1\right)\right) \\
& -\exp \left(\frac{2 \kappa_{I_{\text {agg }}}(1)^{2}}{\kappa_{I_{\text {agg }}}(2)}\right)\left(1-Q\left(-\sqrt{\frac{\kappa_{I_{\mathrm{agg}}}(1)^{3}}{\kappa_{I_{\mathrm{agg}}}(2) z}}\left(\frac{z}{\kappa_{I_{\mathrm{agg}}}(1)}+1\right)\right)\right) .
\end{aligned}
$$

Lognormal distribution: The CCDF of Lognormal distribution is [19]

$$
\operatorname{Pr}\left(I_{\text {agg }}>z\right)^{\operatorname{lognormal}}=Q\left(\frac{\ln z-\kappa_{I_{\text {agg }}}(1)}{\sqrt{\kappa_{I_{\text {agg }}}(2)}}\right) .
$$

where $\ln (\cdot)$ is the natural logarithm.

Shifted lognormal distribution: The CCDF of shifted lognormal distribution can be approximated by [21]

$$
\operatorname{Pr}\left(I_{\text {agg }}>z\right)^{\text {shifted lognormal }} \approx Q\left(\frac{\ln (z-c)-\mu}{\sigma}\right),
$$

where

$$
\begin{aligned}
\sigma^{2} & =\ln \left(\left(k+\sqrt{k^{2}-1}\right)^{\frac{1}{3}}+\left(k-\sqrt{k^{2}-1}\right)^{\frac{1}{3}}-1\right) \\
\mu & =\frac{\ln \left(\frac{\kappa_{I_{\text {agg }}(2)}}{\exp \left(\sigma^{2}\right)-1}\right)-\sigma^{2}}{2} \\
c & =\kappa_{I_{\mathrm{agg}}}(1)-\exp \left(\mu+\frac{\sigma^{2}}{2}\right) \\
k & =1+\beta_{1}\left(I_{\mathrm{agg}}\right)^{2} / 2
\end{aligned}
$$

where $\beta_{1}(\cdot)$ is defined in (15).

Gamma distribution: The CCDF of Gamma distribution is [13]

$$
\operatorname{Pr}\left(I_{\mathrm{agg}}>z\right)^{\mathrm{Gamma}}=1-\frac{\Gamma\left[\frac{\kappa_{I_{\mathrm{agg}}(1)^{2}}}{\kappa_{I_{\mathrm{agg}}}(2)}, 0, \frac{\kappa_{I_{\mathrm{agg}}}(1)}{\kappa_{I_{\mathrm{agg}}}(2)} z\right]}{\Gamma\left[\frac{\kappa_{I_{\mathrm{agg}}}(1)^{2}}{\kappa_{I_{\mathrm{agg}}}(2)}\right]},
$$

where $\Gamma[x]$ and $\Gamma[x, a, b]$ are the complete and incomplete gamma functions, respectively. 


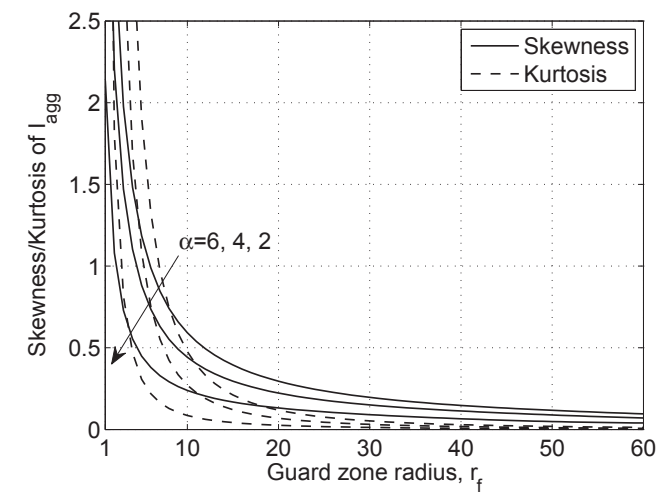

Fig. 2. Skewness and Kurtosis of the aggregate interference versus the radius of guard zone, $r_{f}$ for different path-loss exponents $(\alpha=2,4,6)$ for the case that $\mathrm{PU}$ is located at the center of a disk region with radius $W=100$, $M=1000$ SUs and the Nakagami- $m$ fading channels $(m=4)$.

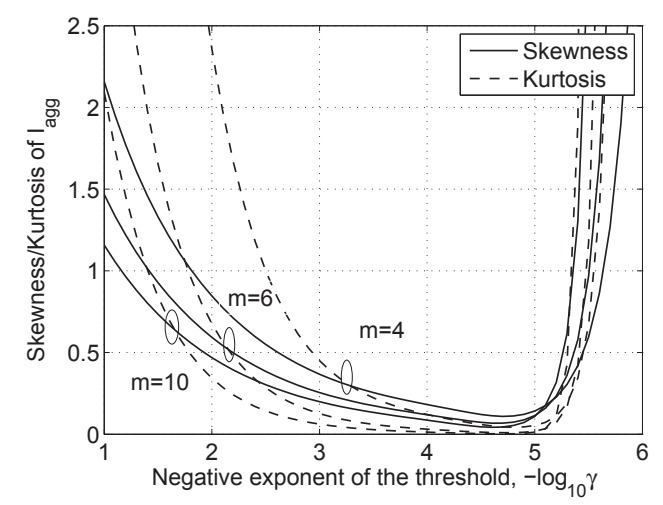

Fig. 3. Skewness and Kurtosis of the aggregate interference versus the negative exponent of the threshold, $-\log _{10} \gamma$ for different Nakagami- $m$ fading parameters $(m=4,6,10)$ for the case that PU is located at the center of a disk region with radius $W=100, M=1000$ SUs and the path-loss exponent $\alpha=2.5$.

\section{RESULTS}

In this section, we present numerical and simulation results to characterize the aggregate interference. The simulation results are generated by uniformly distributing $M$ nodes inside the considered regions over 10 million simulation runs.

\section{A. Convergence to a Gaussian}

First, we investigate the effect of the channel parameters on the convergence of $I_{\text {agg }}$ to a Gaussian distribution using skewness and kurtosis. We consider the scenario that the PU is located at the center of a disk region with radius $W=100$, $M=1000$ SUs and all channels experience Nakagami-m fading.

Fig. 2 plots the skewness and kurtosis of $I_{\text {agg }}$ versus the radius of guard zone, $r_{f}$, for the guard zone protocol for different path-loss exponents $(\alpha=2,4,6)$ and $m=4$. We can see from Fig. 2 that curves are monotonic for the considered range of $r_{f}$. With smaller $\alpha$, skewness and kurtosis converge to 0 at a faster rate.
Fig. 3 shows the skewness and kurtosis of $I_{\text {agg }}$ versus the negative exponent of the threshold, $-\log _{10} \gamma$, for the single-threshold protocol for different fading parameters ( $m=$ $4,6,10$ ) and $\alpha=2.5$. We can see that a larger $m$ (less severe fading) leads to a faster convergence to a Gaussian. However, the curves are not monotonic. Hence, caution must be undertaken in approximating $I_{\text {agg }}$ as a Gaussian, even for a large number of SUs (i.e., $M=1000$ considered in Fig. 3).

\section{B. CCDF Approximation of the Aggregate Interference}

In this section, we compare the different CCDF approximation methods. We consider the following two scenarios: (i) a square region with side length 100 and the PU is located at the corner of this region; (ii) a polygon region as depicted in Fig. 1 with side lengths $S_{1}=200 \sqrt{2 / 3}, S_{2}=200 / \sqrt{3}$ and $S_{3}=S_{4}=S_{1} / 2$ and the PU is located at the intersection point of the two diagonals. All the communication channels undergo the Nakagami- $m(m=4)$ fading and path-loss exponent is $\alpha=3.5$. The radius of the guard zone range is $r_{f}=30$, the threshold for the single-threshold protocol is $\gamma=10^{-5}$ and transmit power for SU is $P_{T}=1$ for these two portocols. For the multi-threshold-protocol, we assume three power levels with $\left(\gamma_{1}=10^{-5}, P_{(T, 1)}=1\right),\left(\gamma_{2}=10^{-3}, P_{(T, 2)}=0.1\right)$, $\left(\gamma_{3}=\infty, P_{(T, 3)}=0\right)$.

Fig. 4 and Fig. 5 plot the approximate CCDF of $I_{\text {agg }}$ for scenario (i) and (ii), respectively. The analytical results are plotted using (10), (13) and (7), along with (17) for Gaussian distribution, (18) for inverse Gaussian, (19) for lognormal distribution, (20) for shifted lognormal distribution and (22) for Gamma distribution.

Comparing Fig. 4(a) and Fig. 5(a), we can see that the shifted lognormal and Gamma distributions both provide good approximations for the guard zone protocol. Comparing Fig. 4(b) and Fig. 5(b), and Fig. 4(c) and Fig. 5(c), we can see that the shifted lognormal distribution provides the best match for the single-threshold and multi-threshold protocols in the distribution tail region. Overall, our results show that the shifted lognormal distribution provides the best CCDF approximation for the considered SU activity protocols and arbitrarily-shaped regions.

\section{CONCLUSIONS}

In this paper, we have studied the aggregate interference inside an arbitrarily-shaped underlay cognitive network region with an arbitrary location of PU and different SU activity protocols. Using the MGF of the interference from a random SU, we have derived the $n$-th moment and the $n$-th cumulant of the aggregate interference from the SUs. Using the cumulants, we studied the aggregate interference's convergence to a Gaussian distribution. In addition, we compared the different distributions which can be used to approximate the distribution of aggregate interference using the exact cumulants. Our results showed that the shifted lognormal distribution provides the best CCDF approximation, especially in the distribution tail region, compared to other distributions considered in the literature. 


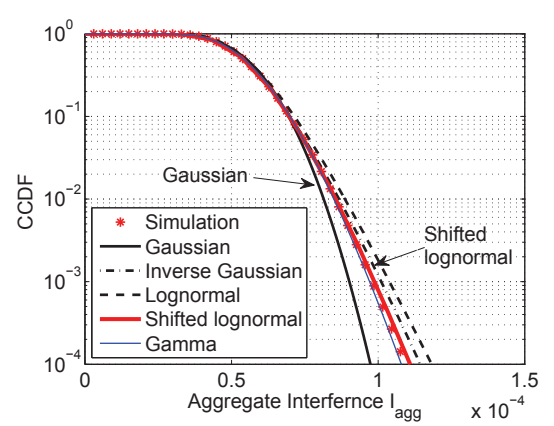

(a) Guard zone protocol $\left(r_{f}=30, P_{T}=1\right)$.

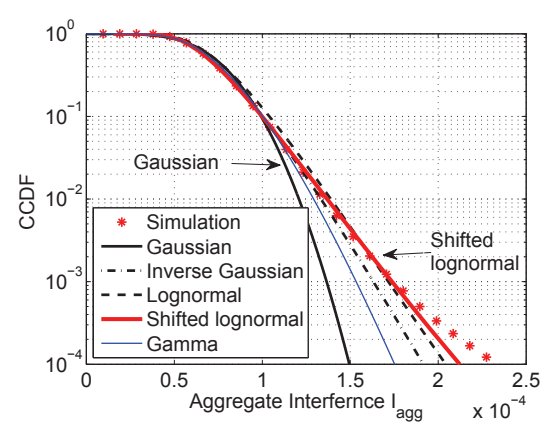

(b) Single-threshold protocol $\left(\gamma=10^{-5}, P_{T}=1\right)$

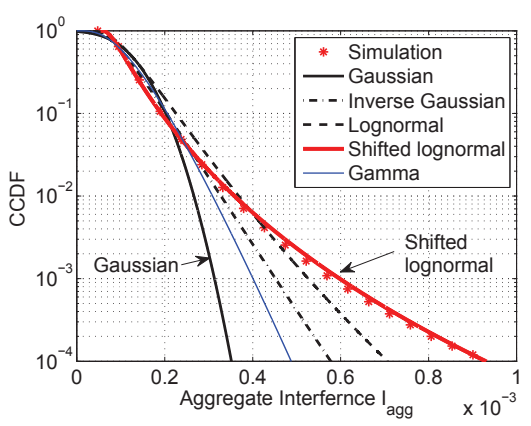

(c) Multi-threshold protocol $\left(\gamma_{1}=10^{-5}, P_{(T, 1)}=\right.$ 1), $\left(\gamma_{2}=10^{-3}, P_{(T, 2)}=0.1\right),\left(\gamma_{3}=\right.$ $\left.\infty, P_{(T, 3)}=0\right)$.

Fig. 4. CCDF approximation for the case that PU is located at the corner of a square region with side length 100, Nakagami- $m(m=4)$ fading and path-loss exponent $\alpha=3.5$.

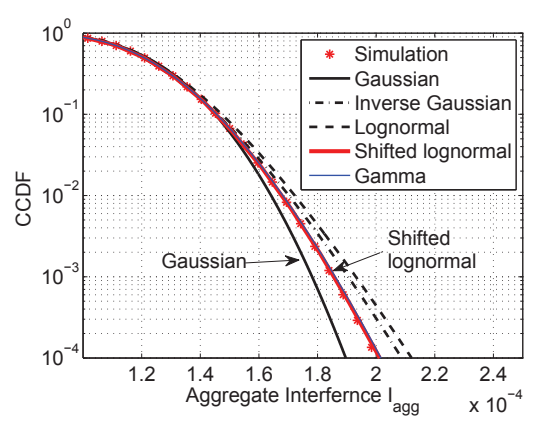

(a) Guard zone protocol $\left(r_{f}=30, P_{T}=1\right)$.

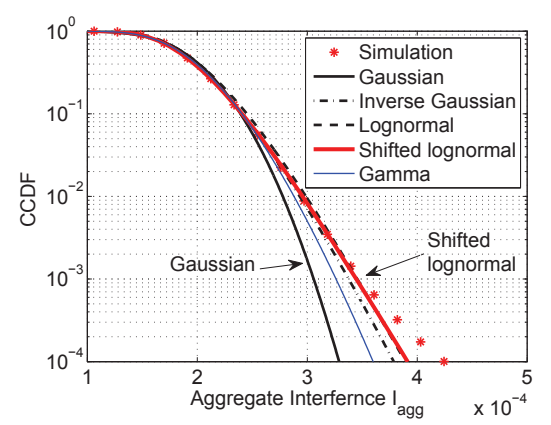

(b) Single-threshold protocol $\left(\gamma=10^{-5}, P_{T}=1\right)$

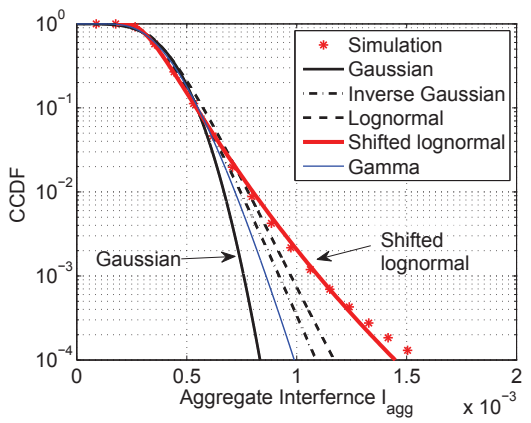

(c) Multi-threshold protocol $\left(\gamma_{1}=10^{-5}, P_{(T, 1)}=\right.$ 1), $\left(\gamma_{2}=10^{-3}, P_{(T, 2)}=0.1\right),\left(\gamma_{3}=\right.$ $\left.\infty, P_{(T, 3)}=0\right)$.

Fig. 5. CCDF approximation for the case that PU is located at the intersection of two diagonals of a polygon region as depicted in Fig. 1, Nakagami- $m$ $(m=4)$ fading and path-loss exponent $\alpha=3.5$.

\section{REFERENCES}

[1] C.-X. Wang, F. Haider, X. Gao, X.-H. You, Y. Yang, D. Yuan, H. Aggoune, H. Haas, S. Fletcher, and E. Hepsaydir, "Cellular architecture and key technologies for $5 \mathrm{G}$ wireless communication networks," IEEE Commun. Mag., vol. 52, no. 2, pp. 122-130, Feb. 2014.

[2] I. F. Akyildiz, W.-Y. Lee, M. C. Vuran, and S. Mohanty, "Next generation/dynamic spectrum access/cognitive radio wireless networks: A survey," Comput. Netw., vol. 50, no. 13, pp. 2127-2159, Sep. 2006.

[3] A. Goldsmith, S. A. Jafar, I. Maric, and S. Srinivasa, "Breaking spectrum gridlock with cognitive radios: An information theoretic perspective,' Proceedings of the IEEE, vol. 97, no. 5, pp. 894-914, May 2009.

[4] C. han Lee and M. Haenggi, "Interference and outage in Poisson cognitive networks," IEEE Trans. Wireless Commun., vol. 11, no. 4, pp. 1392-1401, Apr. 2012.

[5] N. Mahmood, F. Yilmaz, M. Alouini, and G. Oien, "Cognitive interference modeling with applications in power and admission control," in Proc. IEEE DYSPAN, Oct. 2012, pp. 434-439.

[6] A. Rabbachin, T. Quek, H. Shin, and M. Win, "Cognitive network interference," IEEE J. Sel. Areas Commun., vol. 29, no. 2, pp. 480493, Jan. 2011.

[7] L. Vijayandran, P. Dharmawansa, T. Ekman, and C. Tellambura, "Analysis of aggregate interference and primary system performance in finite area cognitive radio networks," IEEE Trans. Commun., vol. 60, no. 7 , pp. 1811-1822, Jul. 2012.

[8] H. ElSawy, E. Hossain, and M. Haenggi, "Stochastic geometry for modeling, analysis, and design of multi-tier and cognitive cellular wireless networks: A survey," IEEE Commun. Surveys \& Tutorials, vol. 15, no. 3, pp. 996-1019, Jul. 2013.

[9] S. Srinivasa and M. Haenggi, "Modeling interference in finite uniformly random networks," in Proc. IEEE ITW, Jun. 2007.
[10] M. Haenggi and R. K. Ganti, Interference in Large Wireless Networks. NOW: Foundations and Trends in Networking, 2009.

[11] R. Menon, R. M. Buehrer, and J. H. Reed, "Impact of exclusion region and spreading in spectrum-sharing ad hoc networks," in Proc. 1st Int. Workshop on Technology and Policy for Accessing Spectrum, Aug. 2006.

[12] A. Ghasemi and E. Sousa, "Interference aggregation in spectrum-sensing cognitive wireless networks," IEEE J. Sel. Topics Signal Process., vol. 2, no. 1 , pp. 41-56, Feb. 2008.

[13] M. Derakhshani and T. Le-Ngoc, "Aggregate interference and capacityoutage analysis in a cognitive radio network," IEEE Trans. Veh. Technol., vol. 61, no. 1, pp. 196-207, Jan. 2012.

[14] Z. Khalid and S. Durrani, "Distance distributions in regular polygons," IEEE Trans. Veh. Technol., vol. 62, no. 5, pp. 2363-2368, Jun. 2013.

[15] J. Guo, S. Durrani, and X. Zhou, "Outage probability in arbitrarilyshaped finite wireless networks," IEEE Trans. Commun., vol. 62, no. 2 pp. 699-712, Feb. 2014.

[16] S. Durrani, J. Guo, and Z. Khalid, "Mathematica and Matlab software for computing distance distributions," http://users.cecs.anu.edu.au/ Salman. Durrani/software.html, May 2013.

[17] M. Haenggi, Stochastic Geometry for Wireless Networks. Cambridge University Press, 2012

[18] J. Guo, S. Durrani, and X. Zhou, "Performance analysis of arbitrarily-shaped underlay cognitive networks: Effect of secondary user activity protocols," 2014, submitted. [Online]. Available: http: //arxiv.org/abs/1403.4669

[19] R. V. Hogg and A. T. Craig, Introduction to Mathematical Statistics, 6th ed. Pearson, 2004.

[20] L. DeCarlo, "On the meaning and use of kurtosis," Psychol. Methods, vol. 2, no. 3, pp. 292-307, 1997.

[21] K. L. Q. Read, "A lognormal approximation for the collectors problem," Amer. Statist., vol. 52, no. 2, pp. 175-180, May 1998. 